BENEDEK, András

\title{
More Visual Content into Vocational Education
}

\section{Introduction - a Chance for a Synthesis}

Quo Vadis Visual Learning? This question probably preoccupies the initiators and organizers of the Visual Learning Lab (VLL), who may approach the question from various aspects, yet in the same way. The VLL monthly research seminars were launched in 2009. As an excellent illustration of the multidisciplinary attitude, they touched on a great variety of topics, and soon led to a series of yearly international conferences, at which by 2014 altogether some 150-200 participants have become "regulars". Our professional diversification is obvious, as illustrated by the VLL homepage http://vll.mpt.bme.hu. The undisputed complexity of the topic is a valid argument. As we were getting along with the story, the over 30 thematic research seminar discussions revealed an exciting pageant where subjective dispositions are supported by disciplinary motives. Budapest University of Technology and Economics (BME), as a classic institution of higher education, added its conservatism, strictly in the positive sense, as a feature related to both technical development and training and education processes. At one of our very first research seminar meetings the topic was "Images, Science, and Higher Education", the discussion referring to books by Martin Kemp ${ }^{1}$ and James Elkins ${ }^{2}$. Soon, too, we succeeded in establishing working connections to a similar earlier - UK initiative. ${ }^{3}$ - Initially the Budapest VLL project perhaps seemed to be nothing more than an example of a small country making a spiritual effort that might even gain some recognition due to its global context; however, the annual international conferences, with the first one organized in 2010, created a new, even more essential scientific debate forum the results of which to date have been published in four volumes of the VISUAL LEARNING series. ${ }^{4}$ Acknowledged international experts who are able to demonstrate the multidisciplinary aspect of the subject in a wider context shared their thoughts and their newest achievements in theoretical and empirical research related to visual tools, directly with the participants of the conferences and indirectly with the broad professional public. An excellent example of this is our long-term collaboration with James E. Katz, an expert of world renown on the social science of mobile communications. ${ }^{5}$

\footnotetext{
${ }^{1}$ Martin Kemp, Visualizations: The Nature Book of Art and Science, Oxford: Oxford University Press, 2000.

2 James Elkins (ed.), Visual Practices Across the University, München: Wilhelm Fink, 2007.

${ }^{3} \mathrm{Cf}$. http://www.nottingham.ac.uk/visuallearninglab/visuallearninglab.aspx, Showcase of Innovations in Visual Learning. Nottingham University, 2010.

${ }^{4}$ András Benedek and Kristóf Nyíri (eds.), Images in Language: Metaphors and Metamorphoses (series VISUAL LEARNING, vol. 1), Frankfurt/M: Peter Lang, 2011, 205 pp. - András Benedek and Kristóf Nyíri (eds.), The Iconic Turn in Education (series VISUAL LeARning, vol. 2), Frankfurt/M.: Peter Lang, 2012, 164 pp. - András Benedek and Kristóf Nyíri (eds.), How to Do Things with Pictures (series VISUAL LeARnING, vol. 3), Frankfurt/M.: Peter Lang, 2013, 224 pp. - András Benedek and Kristóf Nyíri (eds.), The Power of the Image: Emotion, Expression, Explanation (series VISUAL LEARNING, vol. 4), Frankfurt/M.: Peter Lang, 2014, 287 pp.

${ }^{5}$ His latest edited book is the volume Living Inside Mobile Social Information, Division of Emerging Media Studies, College of Communication, Boston University, http://us8.listmanage.com/track/click?u=2fe8f203b1eb854924a38e031\&id=a91e508b8c\&e=94aa0f2163.
} 


\section{Visual Learning - Paradox or Contradiction?}

This paper focusses on a contradiction related to the subject both in an institutional and a broader context and considers a critical element of this process rightfully perceived as progressive. The contradiction roots in the fact that while several disciplines acknowledge the role of visual tools in human communication and it is strongly promoted these days by mobile ICT tools that facilitate visual communication in space and time, this progressive approach could not yet penetrate the theory and practice of education and training.

Also referring to the title of the conference, this paradox of the development of visual learning may be perceived as a parabolic situation whose theoretical and partially practical analysis is particularly important, with special regard to the aspects above. Obviously, we do not have to convince those directly involved in visual learning that images have always had a significant role in human communication. Around the turn of the millennium what we might regard as a kind of visual homecoming had an increasingly strong impact on daily communication, and through that on education, ${ }^{6}$ institutionalized education in particular. The mass penetration of ICT tools brought a significant spontaneity into learning processes; no wonder it was very hard to make it compatible with the visual contents of curricula designed and objectivized in algorithms that had been in turn designed in the framework of the national curricula. The visual contents in traditional textbooks and online curricula, the latter also serving as an illustration of the actual status of the technical infrastructure, have not changed much in the past decades.

A reason behind this phenomenon is conservatism, considered an essential feature of education and knowledge transfer in general that prefers stable, fixed knowledge elements and has had validation, authority and professionalization in the center of its paradigm for centuries, hence being unable to provide the necessary space for visual learning.

\section{Determination of the Issue in the Context of Cultural History}

Visual learning was already known in ancient societies and not only as a tool for daily communication or daily knowledge transfer. Parables created a virtual double dimension, where short, figurative speeches could convey the meaning of an idea by using a picture or metaphor of ordinary life. In European cultural history, the Greek 'parabolé' refers to this. Thus, the word "parable" may be used as an umbrella term that includes every relevant thing from simple comparison to artistically elaborated allegories.

Teaching as a term also has a Biblical interpretation. In particular in the Jewish-Christian tradition, teaching by means of parables used to be immensely popular and the method always generated attention both in Jewish and non-Jewish audiences. Jesus was particularly keen on teaching people this way. Good examples are the Parable of the Lost Son or the Parable of the Good Samaritan where messages are conveyed by means of detailed stories. Obviously, these metaphorical stories were used on purpose, especially as Jesus had to acquit himself from the charge of performing his miracles by the help of Beelzebub. This is how parables had become a consciously applied method of teaching for Jesus, who had to

\footnotetext{
${ }^{6}$ Cf. e.g. Kristóf Nyíri, "Images in Conservative Education”, in Nyíri, Meaning and Motoricity: Essays on Image and Time, Frankfurt/M.: Peter Lang, 2014.
} 
withstand the increasing hatred of the Pharisees who hoped to catch him by something he said so that they could arrest him.

We also have to mention here that 'parabola', i.e. the mathematical interpretation of the word parable and the relevant graphical representations are as old as the stories referred above. The similarity between the mathematical representations and the general features of the communication typical for learning situations is believed not to be incidental. ${ }^{7}$

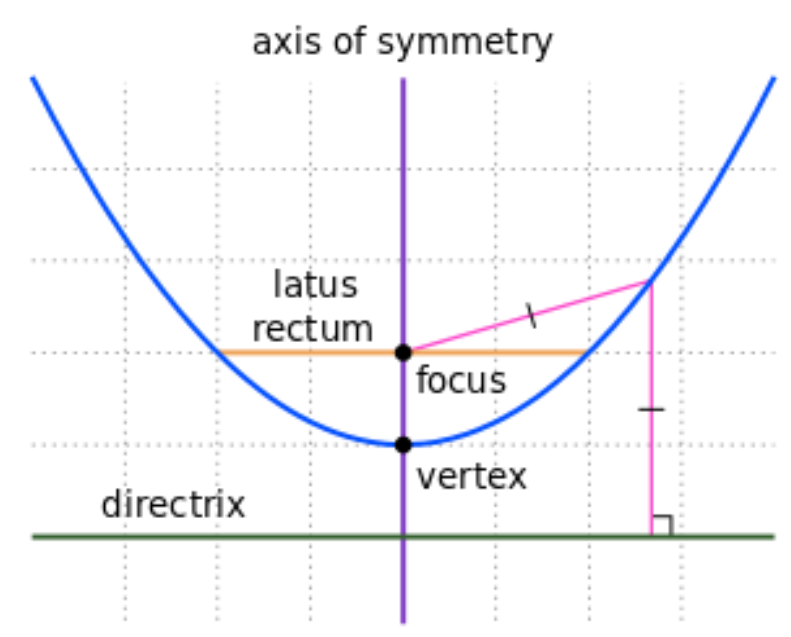

Figure 1

It is important to note before further elaborating the idea that a parabola may be positioned in a Cartesian coordinate plane in a way that its axis of symmetry is represented by the $y$ axis (the axis of ordinates) and its focus is the origo, where the axes of the coordinate system intersect. This construction allows the analysis of correlations where the amount of verbal information is plotted on the horizontal axis and that of visual information on the vertical one.

To provide an illustration for the thoughts to follow, let's take a look at the simplest quadratic function, $f(x)=x^{2}$ and the relevant equation. The equation $y=x^{2}$ does not only determine the form of the curve, but also refers to the nature and ratio of verbal and visual information provided in the curriculum for a particular learning content (hereinafter referred to as $\boldsymbol{T}$, after the Hungarian for knowledge element). The amount of verbal information is plotted on the $\boldsymbol{x}$ axis while that of visual information on the $\boldsymbol{y}$ axis. In our thought experiment, we presume that increasing the amount of verbal information in a linear fashion results in a significant, quadratic increase in the amount of visual information for the same knowledge element $(\boldsymbol{T})$. This presumption may be considered realistic, knowing the usual text to pictures ratio in books, etc. On the other hand, it also indicates that a significant (4fold or even 16-fold) increase in the volume of visual information only corresponds to a 2fold of 4-fold increase in that of verbal information for any given knowledge element on the parabola.

This example emphasizes that according to the equation of the parabola, for the same "directrix sections" (in our example, amounts of verbal information related to a given knowledge element) significantly more visual information is allocated (in our example, the increase is quadratic). If these ratios are altered significantly, the resulting curriculum will be predominantly verbal or predominantly visual. In both cases, acquiring knowledge will

\footnotetext{
${ }^{7}$ Cf. http://en.wikipedia.org/wiki/Parabola (see Figure 1).
} 
probably be severely hindered. We have to note here, that in our thought experiment the ratio of verbal and visual information for a given curriculum is only based on estimations. Though these ratios may appear to be perceivable, calculating the actual functions requires empirical studies.

\section{The Background of this Research}

The creation of open curricula with rich visual contents has become an important direction for the international development of contents and didactics; the process is typically characterized by the constructive contribution by learners. Another typical feature is broad scale public access that is facilitated by modern, interactive on-line interfaces. This specific field is dominated by initiatives in tertiary education (MOOC - Massive Open On-line Courses); however, the significant number of learners in vocational education, the wide range of professions they represent and the fact that the age of those attending such courses has been steadily increasing urge the adaptation of these methods in vocational education as well.

In Hungary, the legislation on public education and vocational training was transformed between 2011 and 2013 and the education of teachers took a new turn. A general expectation regarding the education of teachers is that qualified teachers should be well prepared and able to cope with the following tasks:

- tasks of public education as defined by the National Curriculum according to development fields and educational objectives; transfer of the values and contents of education; knowledge building; development of core competencies and making use of them;

- providing teaching services in public education institutions in accordance with their professional qualifications, in the phases of formal education defined by the Act on Public Education and in the framework of approved curricula based on the National Curriculum; providing teaching services in non-formal education and adult education; performing educational activities in the employing institution;

- creative participation in educational development programs relying on their knowledge and experience.

Thus, our research on didactics essentially focusses on the differentiated control of the work of vocational teachers in class and the application of efficient educational methods and processes. From the aspect of this research, the general criteria of preparing teachers are partly traditional, manifested in knowledge, abilities and attitudes, and partly related to expected teacher competencies, broken down according to the following fields of competences:

- knowledge related to didactics and the given subject;

- supporting, organizing and controlling learning.

Another specific feature of the activity of vocational teachers is that they are allowed to teach not only in schools but also in non-formal vocational training. Hence, they have to be well prepared to teach age groups well outside the range of 14 to 18 year olds, when providing vocational training for young adults and adults or learners with special needs. Training teachers for teaching trade groups or using vocational contents related to particular sectors is hindered by the almost complete lack of textbooks that could support the teaching of professional subjects in vocational schools and vocational secondary schools. Thus, 
teachers could only rely on the actual course requirements within vocational framework curricula. It justifies the need for putting content development for actual subjects and thus the development of a new didactics into the focus of educational development, facilitating both the training of vocational teachers and learning professions at school.

BME is a leading institution in Hungary in the training of vocational teachers and the continuous professional development of teachers, with excellent professional references. Teachers have been trained at BME for more than one and a half centuries. In the past 50 years, significant efforts have been made in the institution as well as at the Department of Technical Education to train vocational teachers, always taking care for also making the relevant developments in didactics. Based on these, BME's Teachers' Training Centre started its operation in December 2012, according to the requirements of the Act on Higher Education, in the framework of BME's Faculty of Economic and Social Sciences (GTK) and continuing the work of the Department of Technical Education.

\section{New Approaches to Research}

The in situ analysis of the history of the issue as given above and the interpretation of parabolas in communication situations led to tackling the practical problems of visual learning from the aspect of education. Improving the efficiency of an education system predominantly relying on verbal contents may represent quite a challenge due to the lack of the necessary time and information and the slowness of correction mechanisms in the case of traditional curricula, as it was proved by several methodology experiments (cooperative methods, project based work, connectivism ${ }^{8}$ in learning). Even "modern" curricula that had been developed by the end of the $20^{\text {th }}$ century had a linear structure and the prevailing dominance of verbal contents ( $80 \%$ on average) was changing only slowly, giving way to visual contents which in turn were mostly composed of static pictures. Though online curricula and multimedia-based e-learning representations include more dynamic visual contents (flash, video), the "logic" of curricula design has not changed in our opinion. Visual contents are still considered as mere supplements to verbal (written and oral) messages.

An alternative to the curriculum design paradigm drafted above may be increasing the ratio of visual elements in order to increase the volume of information. However, the ratio cannot be altered just randomly. Changes should fit into the parabolic equations, assuming that both formats (verbal and visual) are required by learners and their ratio is the same for a given subject, age group and other conditions.

The best example may be the most widely known parabolic function, where the amount of verbal information is plotted on axis $x$ while the amount of visual information (that is able to convey significantly more information) is plotted on axis $y$. These are able to define a focus $(\boldsymbol{F})$ both in theory and reality, corresponding to a relevant knowledge element $(\boldsymbol{T} \boldsymbol{f})$ as discussed previously.

Regarding methodological questions, with special regard to mathematics and other disciplines of natural sciences and the related applied sciences such as technical sciences and their applications, curricula are based on descriptive verbal elements (Text $-\boldsymbol{V}$ ) that are supported by visual elements (Pictures $-\boldsymbol{P}$ ) and mathematical formulas (Math - M).

\footnotetext{
${ }^{8}$ G. Siemens, “Connectivism: A learning theory for the digital age”, International Journal of Instructional Technology and Distance Learning, 2005, http://www.itdl.org/journal/jan 05/article01.htm.
} 
Traditional curricula (published in the format of textbooks) usually include the combination of these, structured in a rigid linear sequence, such as 'explanation, figures, formulation, explanation' and so on. Often only random examples are given as case studies (Case $-\boldsymbol{C}$ ) to illustrate practical applications. Even tests to facilitate practicing follow this structure or they are provided in a sequenced order.

- In the framework of our ongoing research to develop new curriculum designs, we use online tools and methods to present knowledge elements $\left(T_{f 1} \ldots T_{f n}\right)$ in a virtual space instead of the traditional sequenced order (that obviously used to be also a prerequisite for printing). In this virtual space, the $T_{f 1} \ldots T_{f n}$ knowledge elements are placed on the surface of a sphere and positions are not defined by moving them but adding more and more elements. The visual representation applied for the virtual encyclopedia clearly demonstrates the connections between the individual entries that create a system similar to a scale-independent network. An example of this method is the Magyar Virtuális Enciklopédia (Hungarian Virtual Encyclopedia, http://www.hunfi.hu/nyiri/enc), a result of a development process realized decades ago that is still able to demonstrate the success of the method.

- For a curriculum design developed in open access, cloud services offer a development infrastructure surpassing all previous solutions. This far we used series like

$$
\boldsymbol{T}-\boldsymbol{P}-\boldsymbol{M}
$$

sometimes supported with practical examples

$$
\boldsymbol{T}-\boldsymbol{P}-\boldsymbol{M}-\boldsymbol{C}
$$

Showing other connections between these elements to develop a dynamic network was typically hindered by disciplinary and temporal restrictions.

Basing on our current development and the relevant hypothesis, the new curriculum may greatly facilitate the acquisition of knowledge and its control. This new curriculum, where verbal and visual elements are presented in a one to one ratio and knowledge elements are organized into a network, would be scale-independent and structured as a graph; it would also be supported by a mathematical representation to enhance both its theoretical and practical aspects and users would be allowed to extend it by case studies and practical examples. For each element of this knowledge set formulated in the virtual space in a graph structure, a comics would be assigned, comics being a solution that combines verbal and visual information as well as being parallelled by a mathematical representation and adjustable for the needs of the given age group. These knowledge elements, to be verified upon a broad professional basis, would basically summarise theoretical and general information in a modern form, yet in an optimum volume. As a result of the open structure, the new system is obviously more suitable for incorporating new and interdisciplinary knowledge elements than traditional solutions. Another important characteristic of this knowledge representation process is increasing the efficiency of acquiring knowledge by means of making dry theoretical knowledge more lifelike adding case studies, good practices and examples to it which in turn are the results of social content development. The parable of visual learning becomes reality at this point. The verbal dominance in the current traditional education framework cannot be overcome due to reasons like tradition, methodology culture, generations of textbooks, whereas in the new system the $\boldsymbol{T} \boldsymbol{P} \boldsymbol{P}-\boldsymbol{M}$ knowledge structure, originally restricted both in space and "volume" may be supported by a $\boldsymbol{C}$ set of case studies unlimited regarding the number and nature of examples, where practicality and the need for illustration ab ovo presumes the dominance of visual elements. 
Our concept aims at providing a major storage medium by means of cloud services, as needed by the curriculum that is being developed using the open access approach. This way, a development infrastructure superior to any former attempts can be provided for teachers, teacher trainees or even students involved in developing didactics or learning contents in the medium term.

Based on the current developments, our hypothesis states that knowledge elements interlinked at the level of micro-contents will form a complex curriculum. The practical application of micro-contents as micro learning elements is justified by the new habitual ways of gaining information by means of the smart devices generally used now. Common features of the most popular mobile communication applications are the offering of instant experience, direct communication with friends and others using the same application, and customized "quantum" of received knowledge. This customization depends on the size and resolution of the mobile device's screen, the limitations of the user's divided attention, the design of the user interface and the way the user attempts to eliminate environmental disturbance resulting from being in motion while using the device.

Another feature of modern mobile communication is that digital information can be "recycled" that is shared among connected people. There are several mobile applications that support the unlimited accumulation of information (Facebook, Pinterest). This is a fixed use of mobile devices, mostly practiced by the young generations, that appears to be a yet unexploited opportunity in Hungarian education. Micro-learning as such is already known in Hungary from international practical examples (duolingo - learning foreign languages); however, there is no relevant methodology specifically adapted to Hungarian conditions.

Micro-learning presumes the generation of micro-contents and constructing their relevance network. Using these methods, traditional learning contents may be customized in a digital format for the screen of mobile devices and the context of these contents may be displayed for students like a hypertext. Knowledge elements may be collected and copied into sets of contents; these sets may be then analyzed, estimating the quality of the entire curriculum regarding its contents and configuration.

Curricula that can be modelled as a scale-independent graph (that is connected visual and verbal knowledge elements) combined with mathematical representations to enhance both theoretical and practical approaches, as well as case studies and practical examples, also added by the users themselves, may significantly promote acquiring and controlling knowledge. Accordingly, verbal explanations, visual representations easily understood by young people and mathematical representations adjusted to the relevant age group would be supplied to each element of the graph-like knowledge set formulating in virtual space. These knowledge elements, formed on a broad professional base but verifiable by the culture of didactics of teachers would summarize theoretical and general information in a modern format and in optimum quantity. As a result of its open structure, it is easier to add knowledge elements related to new or interdisciplinary contexts in this system than in traditional ones. Other important characteristics of this knowledge representation process are improving the efficiency of acquiring knowledge by applying case studies and examples and accelerating targeted content development by the community (students and teachers). Case studies, best practices and practical examples may render learning and the curriculum itself more realistic, compared to the current, apparently often too theoretical approach.

Another significant feature of the research regarding didactics is the use of cloud based services i.e. storing the complex open access didactics material (that is being expanded continuously regarding both the number of elements and their connections) in a cloud. It is 
thought to provide services for users in schools that are not restricted to one dedicated piece of hardware but to several ones owned by the service provider, guaranteeing the continuous availability of redundant servers combined with an efficient protection against data losses.

Cloud services appeared in the 2010s on the IT market and the number of cloud service providers has been rapidly increasing lately, while state-of-the-art ICT tools facilitate their use for everyday purposes. The most frequently cited advantages of cloud based solutions are reliable and cost efficient implementation and operation, $80-90 \%$ exploitation of the available capacity, low investment costs and unlimited computer resources.

Cloud based systems generally attempt to establish cross links between various services. This is an essential feature of the system as this way a particular service has access to contents generated in another one so users do not have to bother with uploading all the necessary data and information when starting a new application. The most important feature of the open access application principle is free availability where the progressive use of security protocols is not required. Other advantages of this approach are the possibility of collaboration, continuous synchronizing and saving of data, automatic updates, shared contents and encryption.

In cloud based data storage, several data storage providers are available, all of them specialized at using a particular platform, working in full harmony with the given operating system. For example, the possibly most widely used storage providers in global comparison are Google Drive combined with the Android operating system, Dropbox with iOS systems and OneDrive with the increasingly popular Windows phone operating system. The projected rate of increase in cloud based services will exceed that of general IT technologies multiple (four or five) times. This is why curriculum and learning content development at BME focuses on generating visual contents by professional communities (teachers' development programs) when developing vocational contents at BME.

The strategic concept of these developments is being elaborated currently. The first design of this kind is being developed for the new online curriculum "Introduction into Systems" for teachers working in secondary education. Our hypothesis can be summarized as follows: visual learning may provide opportunities to use parables that are able to improve the efficiency of human learning, currently based on traditional verbal communication and as such hindered by time constraints and information pressure.

\section{Conclusion}

The most up-to-date interpretation of the never ceasing debate that was provoked by Ferguson's Engineering and the Mind's Eye almost half a century ago points out how poorly our current education paradigm is able to exploit the potential of visual learning. The historic experiences in interpreting and using parables called the attention to the fact that these two methods of communication do not necessarily contradict each other as educational tools. Indeed, state-of-the-art interpretations of learning make us abandon teaching traditions when designing new curricula and exploit the new technical options offered by our digital environment, providing learners with a flexible, continuously developing system where proven methods are available to extend the set of relevant practical examples. Applying visual learning in the framework of this approach, we may fully utilize its potentials in 
creating opportunities to develop for those who want to hand over knowledge and those who wish to acquire it.

\section{References}

[1] Martin Kemp, Visualizations: The Nature Book of Art and Science, Oxford: Oxford University Press, 2000.

[2] James Elkins (ed.), Visual Practices Across the University, München: Wilhelm Fink, 2007.

[3] Cf. http://www.nottingham.ac.uk/visuallearninglab/visuallearninglab.aspx, Showcase of Innovations in Visual Learning. Nottingham University, 2010.

[4] András Benedek and Kristóf Nyíri (eds.), Images in Language: Metaphors and Metamorphoses (series Visual Learning, vol. 1), Frankfurt/M: Peter Lang, 2011, 205 pp. András Benedek and Kristóf Nyíri (eds.), The Iconic Turn in Education (series Visual Learning, vol. 2), Frankfurt/M.: Peter Lang, 2012, 164 pp. - András Benedek and Kristóf Nyíri (eds.), How to Do Things with Pictures (series Visual Learning, vol. 3), Frankfurt/M.: Peter Lang, 2013, 224 pp. - András Benedek and Kristóf Nyíri (eds.), The Power of the Image: Emotion, Expression, Explanation (series Visual Learning, vol. 4), Frankfurt/M.: Peter Lang, 2014, 287 pp.

[5] His latest edited book is the volume Living Inside Mobile Social Information, Division of Emerging Media Studies, College of Communication, Boston University, http://us8.listmanage.com/track/click? u=2fe8f203b1eb854924a38e031\&id=a91e508b8c\&e=94aa0f2163. [6] Cf. e.g. Kristóf Nyíri, "Images in Conservative Education", in Nyíri, Meaning and Motoricity: Essays on Image and Time, Frankfurt/M.: Peter Lang, 2014.

[7] Cf. http://en.wikipedia.org/wiki/Parabola (see Figure 1).

[8] G. Siemens, "Connectivism: A learning theory for the digital age", International Journal of Instructional Technology and Distance Learning, 2005, http://www.itdl.org/journal/jan_05/article01.htm. 